\title{
Magnetic noise contribution of the ferromagnetic core of induction magnetometers
}

\author{
C. Coillot ${ }^{1}$, M. El Moussalim ${ }^{2}$, E. Brun ${ }^{3}$, A. Rhouni ${ }^{2}$, R. Lebourgeois ${ }^{3}$, G. Sou ${ }^{4}$, and M. Mansour ${ }^{2}$ \\ ${ }^{1}$ BioNanoNMRI-group, Laboratoire Charles Coulomb (L2C), Universite de Montpellier, Place Eugene \\ Bataillon, \\ 34095 Montpellier, France \\ ${ }^{2}$ Laboratoire de Physique des Plasmas (LPP), Ecole Polytechnique, Route de Saclay, 91128 Palaiseau, France \\ ${ }^{3}$ Thales Research and Technology, Palaiseau, France \\ ${ }^{4}$ Laboratoire d'Electronique et d'Electromagnetisme (L2E), Université Pierre et Marie Curie, Paris, France
}

Correspondence to: C. Coillot (christophe.coillot@univ-montp2.fr)

Received: 16 February 2014 - Revised: 5 May 2015 - Accepted: 15 May 2015 - Published: 18 June 2015

\begin{abstract}
The performance of induction magnetometers, in terms of resolution, depends both on the induction sensor and the electronic circuit. To investigate accurately the sensor noise sources, an induction sensor, made of a ferrite ferromagnetic core, is combined with a dedicated low voltage and current noise preamplifier, designed in CMOS $0.35 \mu \mathrm{m}$ technology. A modelling of the contribution of the ferromagnetic core to the noise through the complex permeability formalism is performed. Its comparison with experimental measurements highlight another possible source for the dominating noise near the resonance.
\end{abstract}

\section{Introduction}

Induction magnetometers are used in a wide range of applications (Ripka, 2000; Coillot, 2013) to measure extremely weak magnetic fields over a wide frequency range (from $\mathrm{mHz}$ up to $\mathrm{GHz}$ ). At $1 \mathrm{~Hz}$, for magnetotelluric waves observation purposes, noise equivalent magnetic induction about $0.2 \mathrm{pT} \mathrm{Hz}^{-1}$ is reported in Bin (2013). The context of this work concerns the study and the design of an induction magnetometer in the very low-low frequency (VLF-VF) range to investigate plasma waves in space in Jupiter's environment for an ESA mission. For this purpose, the goal of electromagnetic wave measurement, given in terms of noise equivalent magnetic induction (NEMI in $\mathrm{T} \mathrm{Hz}^{-1}$ ), is challenging. An ability to reach NEMI lower than $10 \mathrm{fT} \mathrm{Hz}^{-1}$ at $10 \mathrm{kHz}$ is mandatory. Due to the severe radiation environment, it has been considered to locate the preamplifier either inside the hollow ferromagnetic core of the induction sensor (Grosz, 2010) or inside the mechanical tri-axis structure to take advantage of an efficient radiation shielding provided by the sensor itself. An ASIC preamplifier designed in $0.35 \mu \mathrm{m}$ CMOS technology (Rhouni, 2012; Ozaki, 2014) offers a possibility of achieving very efficient induction magnetometers. In the context of this work we designed an ASIC low noise preamplifier (called MAGIC2) which offers especially low noise parameters which make possible the investigation of the noise source of the induction magnetometer. This work aims to extend the induction magnetometer modelling presented in Coillot and Leroy (2012) by introducing the noise source arising from the ferromagnetic core based on physical modelling of the complex permeability. Usually this noise source, which is frequency dependent, can be hidden by other dominating noise sources (especially the equivalent input current noise from the preamplifier). However, the use of a low input current noise preamplifier permits one to enhance the noise source from the sensor itself near the resonance. For this purpose, a comparison between the modelling and the measurement of the NEMI is performed on a $12 \mathrm{~cm}$ length sensor using a commercial Mn-Zn ferrite core (3C95 from Ferroxcube) of diabolo shape. 


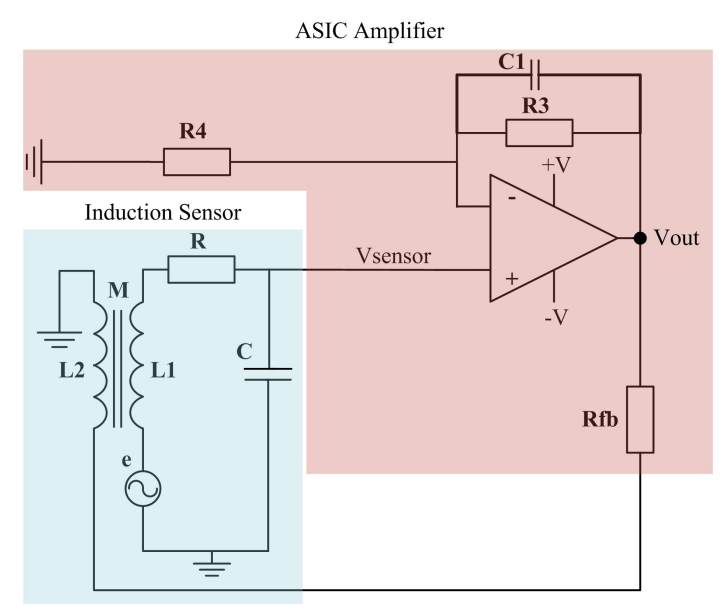

Figure 1. Feedback flux principle.

\section{Induction magnetometer using feedback flux: generalities}

In this section we briefly remind the reader of the basis of an induction magnetometer using feedback flux. Induction sensors are basically built with an $N$ turns coil of section $S$. When the coil is wound around a ferromagnetic core, the induced voltage is multiplied by a factor $\mu_{\text {app }}$ known as apparent permeability (described in Sect. 4.1). In harmonic regime at angular frequency $\omega$, the induction voltage is written as

$e=-j \omega N S \mu_{\mathrm{app}} B$

where $j^{2}=-1$ is the imaginary unit and $B$ is the magnetic flux density to be measured. The electrokinetic modelling assumes that the induced voltage $e$ is in series with the resistance $R$ and the inductance $L$, while the accessible voltage ( $\left.V_{\text {out }}\right)$ is got at the capacitance $C$ terminals. The transmittance of the induction sensor exhibits a resonance at angular frequency $\omega_{0}=1 / \sqrt{ }(L C)$. In order to remove the resonance, two kinds of electronic conditioning are classically implemented: a feedback flux amplifier or a transimpedance amplifier (Tumanski, 2007). In this work, we will focus only on the feedback flux amplifier schematically presented in Fig. 1.

The transmittance of the feedback flux amplifier is expressed as

$T(j \omega)=\frac{V_{\mathrm{OUT}}}{B}=\frac{-j N S G \mu_{\mathrm{app}} \omega}{\left(1-L C \omega^{2}\right)+j \omega\left(R C+\frac{G M}{R_{\mathrm{fb}}}\right)}$,

where $j$ is the unity imaginary number, $G$ is the voltage gain of the amplifier, $M$ is the mutual inductance between the measurement winding and the feedback one and $R_{\mathrm{fb}}$ is the feedback resistance. In the following section we will focus on the ASIC amplifier design and its noise parameters.

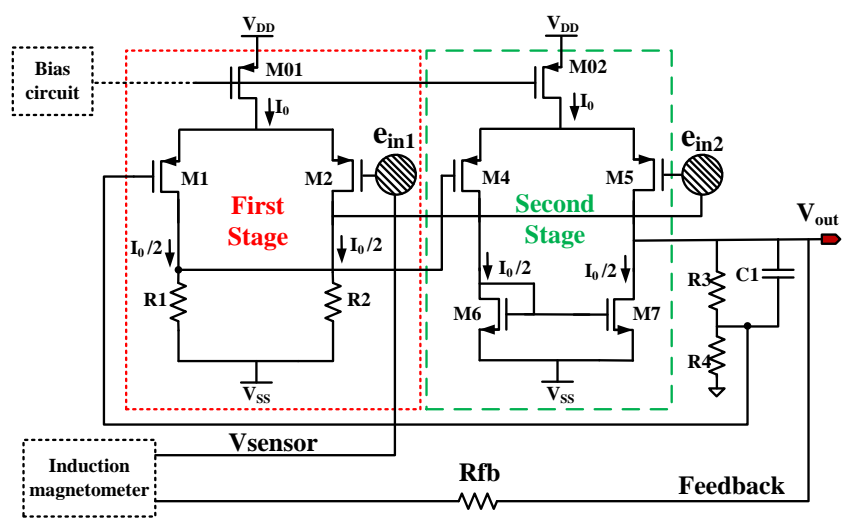

Figure 2. Schematic of the ASIC amplifier design.

\section{Low voltage and current noises ASIC amplifier design for feedback flux induction magnetometers}

To preserve the sensor noise performances in terms of NEMI, the equivalent input voltage noise $\left(e_{\mathrm{PA}}\right)$ and the input current noise $\left(i_{\mathrm{PA}}\right)$ of the amplifier must be as low as possible with a special awareness of $1 / f$ noise. The requirement of the ASIC amplifier design is to satisfy $3 \mathrm{nV} \mathrm{Hz}^{-1}$ of equivalent input voltage noise and a few tens of $\mathrm{fA} \mathrm{Hz}^{-1}$ of equivalent input current noise on a frequency range from $10 \mathrm{kHz}$ up to $1 \mathrm{MHz}$. The gain needs to be about $50 \mathrm{~dB}$ to be suited to the 16 bit ADC and the power consumption should be lower than $30 \mathrm{~mW}$. In this context, CMOS technology, which is mainly composed of MOSFET transistors, is an adequate solution. In the following section, design steps, voltage noise modelling and some measurement results of the low noise ASIC preamplifier are given.

\subsection{Open loop noise considerations}

It is detailed in Rhouni (2012) that, for the same gate size (W/L), the $1 / f$ noise of a PMOS transistor is lower than a NMOS one. To achieve a very low noise performance and a high gain, the amplifier is composed of two stages (Fig. 2): the first stage represents the main contribution to the total output noise, while the second one aims to increase the gain of the open loop amplifier. The first stage is a simple PMOS differential pair with resistive charges $\left(\mathrm{R}_{1}\right.$ and $\left.\mathrm{R}_{2}\right)$. In this configuration the input transistor $\left(\mathrm{M}_{1}\right.$ and $\left.\mathrm{M}_{2}\right)$ design is related to the low input voltage noise performance, while the combination of the drain resistance $\left(\mathrm{R}_{1}\right)$ and the transconductance $g_{\mathrm{m}_{1}}$ of the input transistors $\mathrm{M}_{1}$ and $\mathrm{M}_{2}$ is used to set the gain $(A)$ of the differential pair:

$A=g_{\mathrm{m}_{1}} \mathrm{R}_{1}$.

By considering the thermal noise of the input pair transistor, the low-frequency noise from the input pair transistor and the thermal noise arising from the drain resistance, the 
power spectrum density of the equivalent input noise $\left(e_{\mathrm{in} 1}^{2}\right)$ of the preamplifier's first stage can be obtained:

$e_{\mathrm{in} 1}^{2}=2\left(\frac{8 k T}{3 g_{\mathrm{m}_{1}}}+\frac{K F I_{\mathrm{d} 1}^{\mathrm{AF}}}{C_{o x} L_{1} W_{1} f g_{m_{1}}^{2}}+\frac{4 k T}{g_{\mathrm{m}_{1}}^{2} \mathrm{R}_{1}}\right)$,

where $k$ is the Boltzmann constant, $T$ is the temperature in $K, I_{\mathrm{d} 1}=I_{0} / 2$ is the drain resistance, $W_{1}$ is the channel width, and $L_{1}$ is the gate length of the input transistors. AF $(=1.4)$ and $\mathrm{KF}(=1.8 e-26)$ are PMOS noise parameters. Knowing that $g_{\mathrm{m}_{1}}=\sqrt{2 I_{\mathrm{d} 1} K_{P}^{\prime} \frac{W_{1}}{L_{1}}}$ and $I_{0}=2 I_{\mathrm{d} 1}$, we get

$e_{\mathrm{in} 1}^{2}=\frac{16 k T}{3 \sqrt{I_{0} K_{P}^{\prime} \frac{W_{1}}{L_{1}}}}+\frac{\mathrm{KF} / 2^{\mathrm{AF}}}{K_{P}^{\prime} C_{\mathrm{ox}} W_{1}^{2} f} I_{0}^{\mathrm{AF}-1}+\frac{8 k T}{I_{0} K_{P}^{\prime} \frac{W_{1}}{L_{1}} \mathrm{R}_{1}}$,

where $I_{0}$ is the bias current and $K_{P}^{\prime}=C_{\text {ox }} \mu_{p}$.

As shown in the equation, $e_{\text {in } 1}$ depends on the transistor gate size $\left(W_{1} / L_{1}\right)$ and the bias current $I_{0}$. A trade-off between the size and the power consumption of the final circuit must be specified considering the noise objective. Increasing the transistor size allows one to decrease its $1 / f$ noise contribution. The current is also set to help in reducing the noise regarding the power consumption and the gain. In order to achieve $3 \mathrm{nV} \mathrm{Hz}^{-1}$ at $10 \mathrm{kHz}$ of equivalent input noise and a minimum gain $A_{1}=30 \mathrm{~dB}, I_{0}$ was set to $2 \mathrm{~mA}, W_{1}=$ $W_{2}=5000 \mu \mathrm{m}, L_{1}=L_{2}=1.2 \mu \mathrm{m}$ and $R_{1}=R_{2}=3 \mathrm{k} \Omega$.

The second stage, which is a PMOS differential pair (M4M5) with a NMOS load (M6-M7), will allow one to enhance the open loop gain to achieve the closed loop gain specification $\left(G_{\mathrm{dB}}=50 \mathrm{~dB}\right)$ over the desired frequency bandwidth $(>50 \mathrm{kHz})$. This stage contains a minimum number of transistors to save power consumption, silicon area and specifically the noise performance of the first stage. The power spectrum density of the voltage noise of this stage referred to as M4-M5 input is written as

$$
\begin{aligned}
e_{\mathrm{in} 2}^{2}= & \frac{2 B_{P} I_{0}^{\mathrm{AF}-1}}{W_{5}^{2} f}\left(1+\frac{K_{N}^{\prime} B_{N}}{K_{P}^{\prime} B_{P}} \frac{L_{5} W_{5}}{L_{7}^{2}}\right) \\
& +\frac{16 k T}{3 \sqrt{I_{0} K_{P}^{\prime} \frac{W_{5}}{L_{5}}}}\left(1+\sqrt{\frac{K_{N}^{\prime} W_{7} L_{5}}{K_{P}^{\prime} W_{5} L_{7}}}\right),
\end{aligned}
$$

with $B_{N}=K F /\left(2 K_{N}^{\prime} C_{\text {ox }}\right)$ and $B_{P}=K F /\left(2 K_{P}^{\prime} C_{\text {ox }}\right)$.

The second stage provides a gain $A_{2}=55 \mathrm{~dB}$ if $W_{4}=$ $W_{5}=5000 \mu \mathrm{m}$ and $W_{6}=W_{7}=50 \mu \mathrm{m}$.

The total equivalent input noise voltage $e_{\mathrm{PA}}$ is finally calculated using the PSDs of each stage $e_{\mathrm{in} 1}^{2}$ and $e_{\mathrm{in} 2}^{2}$ and their open loop gains $A_{1}$ and $A_{2}$ :

$e_{\mathrm{PA}}=\sqrt{\frac{e_{\mathrm{out}}^{2}}{A_{1}^{2} A_{2}^{2}}}=\sqrt{\frac{2 A_{1}^{2} A_{2}^{2} e_{\mathrm{in} 1}^{2}+A_{2}^{2} e_{\mathrm{in} 2}^{2}}{A_{1}^{2} A_{2}^{2}}}$.

This last equation can be used to get the noise objective for the second stage, ensuring that $e_{\mathrm{PA}}$ is equal to $3 \mathrm{nV} \mathrm{Hz}^{-1}$ at $10 \mathrm{kHz}$.

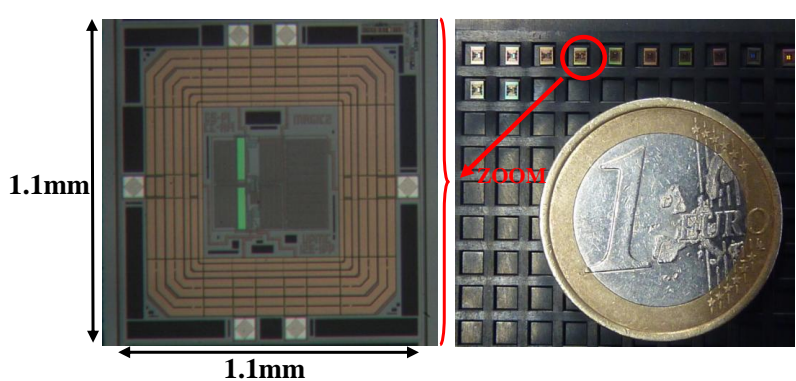

Figure 3. Photographs of the low noise ASIC amplifier named MAGIC2.

\subsection{Closed loop noise considerations}

To make the gain of the amplifier weakly sensitive to temperature variation, the amplifier is used in a closed-loop configuration. The closed-loop gain is set by the $R_{3}$ to $R_{4}$ ratio ( $\left.G=1+R_{3} / R_{4}\right)$, while the $C_{1}$ capacitance is needed to ensure the phase margin. Since this capacitance does not impact the noise analysis, it will not be considered in the rest of the article. As decribed in Sobering (1999), in the context of operational amplifier noise analysis, our noise analysis can be summarized as three contributions: the equivalent voltage input noise at the non-inverting input $\left(e_{\mathrm{PA}}\right)$, the Johnson noise in $R_{4}$ at the inverting input and the Johnson noise in $R_{3}$. According to the Sobering (1999) analysis, two gains should be considered: the inverting $\left(A_{\mathrm{v}-\text { inv }}\right)$ and noninverting ones $\left(A_{\mathrm{v}-\text { non-inv }}\right)$. However, in the case of an ideal op amp (which is a correct hypothesis in our design since the open loop gain is $A_{1}+A_{2}=85 \mathrm{~dB}$ ), these two gains are written $A_{\mathrm{v}-\text { inv }}=R_{3} / R_{4}$ and $A_{\mathrm{v}-\text { non-inv }}=1+R_{3} / R_{4}$. Moreover, in the case of high closed loop gain (i.e. $R_{3} / R_{4} \gg 1$ ), these two gains can be considered to be identical. That allows us to consider the equivalent op amp input noise to be

$e_{\mathrm{OpAmp}}^{2}=e_{\mathrm{PA}}^{2}+4 k T R 4$.

Lastly, the input referred noise contribution coming from gain resistance of the preamplifier $\left(R_{4}\right)$ is neglected, since its value is small (in our design we got $R_{4}=28 \Omega$, which leads to an equivalent voltage noise contribution of about one-tenth of $\left.e_{\mathrm{PA}}\right)$.

\subsection{Measurement results and performances}

The amplifier was fabricated in a standard $0.35 \mu \mathrm{m}$ fourmetal bulk CMOS process. The manufactured circuit has a $1.21 \mathrm{~mm}^{2}$ area. The silicon chip contains one amplifier. Its microphotograph is shown in Fig. 3.

The gain transfer function and the equivalent input noise have been characterized. A high pass filter, with cut-off frequency at $1 \mathrm{~Hz}$, is inserted to remove DC offset, while the low pass filtering cut-off frequency is due to the combination $R_{3}$ and $C_{1}$. Figure 4 shows that the gain is about $50.7 \mathrm{~dB}$ from a few $\mathrm{Hz}$ (a high pass filtering is inserted to remove 


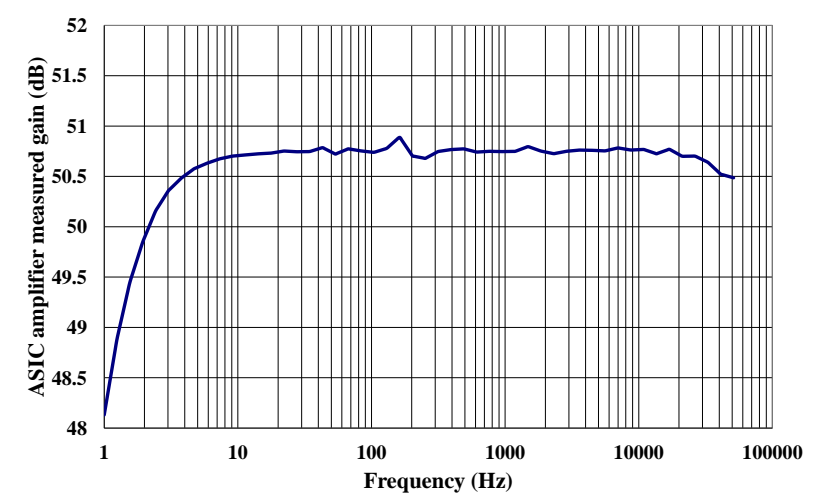

Figure 4. Amplifier transfer function (in dB) of MAGIC2.

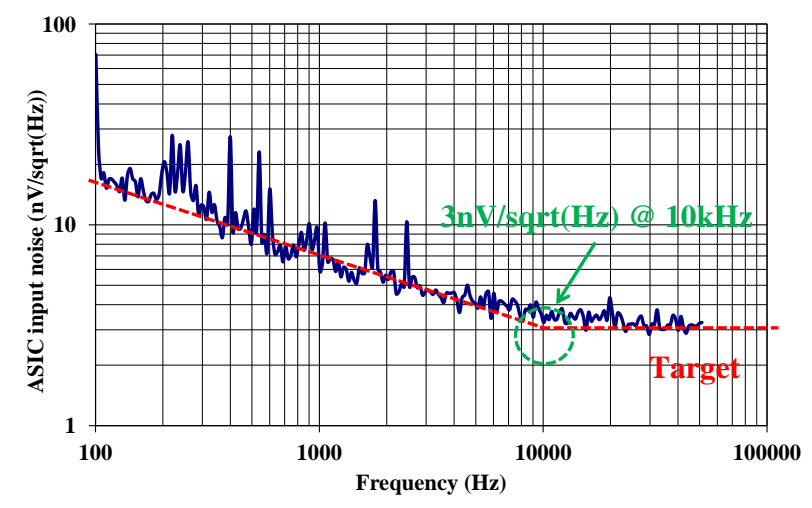

Figure 5. Equivalent input referred noise (in $\mathrm{nV} \sqrt{(\mathrm{Hz})}^{-1}$ ) measured using a $50 \Omega$ input resistor.

the offset) up to $50 \mathrm{kHz}$. The measured gain value is consistent with the $1+R_{3} / R_{4}$ ratio $\left(R_{3}=10 \mathrm{k} \Omega\right.$ and $\left.R_{4}=28 \Omega\right)$. Figure 5 demonstrates a measured input voltage noise about $3 \mathrm{nV} \mathrm{Hz}^{-1}$ at $10 \mathrm{kHz}$ when connecting a $50 \Omega$ resistor at the input of the amplifier.

The induction sensor has a very high input impedance. It implies that it is essential to minimize the input noise current of the amplifier since it will lead to a high contribution to the output noise voltage. In our design, the input current noise contribution is less than $20 \mathrm{fA} \sqrt{\mathrm{Hz}}^{-1}$, which is achieved thanks to the CMOS technology. It can be concluded, for this part, that the combination of low power consumption, low input voltage noise and low current noise, which are essential to induction sensors, can be achieved using CMOS technology at the price of a significant design work.

\section{Modelling of the ferromagnetic core noise source contribution}

The NEMI reaches its minimum value in the decade around the resonance frequency. The usual modelling of the NEMI will underestimate its value in this frequency range. In rare works, to our best knowledge, noise sources related to the ferromagnetic material are evoked either through an empirical correlation (Seran and Fergeau, 2005) or a set of quality factors (Korepanov, 2010) to take into account the NEMI increase near to the resonance. In the first quoted paper, the coefficient of the correlation is determined experimentally for a given core size, which does not allow one to take it into account in a preliminary design stage, while, in the second paper, the quality factor values are given from a tentative estimation. At low ambient field $(<\mathrm{mT})$, the noise in the ferromagnetic core comes either from an eddy current or from magnetization mechanisms like domain wall relaxation and magnetization rotation. At a high magnetic field (typ. mT), Barkhausen noise, related to domain wall jumps, will occur. The usual domain of application of an induction magnetometer is related to a quiet electromagnetic environment; thus, Barkhausen noise will not be considered in this study.

\subsection{Complex permeability of the $\mathrm{Mn}-\mathrm{Zn}$ ferrite}

The mentioned noise source can be modelled through the concept of complex permeability (Tsutaoka, 2003) where the imaginary part of the permeability is related to the ferromagnetic noise source. For high permeability $\mathrm{Mn}-\mathrm{Zn}$ sintered ferrite, we use the complex susceptibility of resonance type given in Dosoudil (2004). The first fraction in the susceptibility relation (Eq. 9) corresponds to the frequency dispersion of domain wall motion contribution, while the second fraction represents the magnetic moment rotation contribution.

$\mu=1+\frac{\omega_{\mathrm{d}}^{2} \chi_{\mathrm{d} 0}}{\omega_{\mathrm{d}}^{2}-\omega^{2}+i \omega \beta}+\frac{\left(\omega_{\mathrm{s}}+j \omega \alpha\right) \omega_{\mathrm{s}} \chi_{\mathrm{s} 0}}{\left(\omega_{\mathrm{s}}+i \omega \alpha\right)^{2}-\omega^{2}}$,

where $\chi_{\mathrm{d} 0}$ and $\chi_{\mathrm{s} 0}$ are the static susceptibilities for domain wall motion and magnetic moment rotation, $\omega_{\mathrm{d}}\left(=2 \pi f_{\mathrm{d}}\right)$ and $\omega_{\mathrm{s}}\left(=2 \pi f_{\mathrm{s}}\right)$ are resonance frequencies of domain wall motion and magnetic moment rotation, $\beta$ and $\alpha$ are the damping factors, and $f=\omega /(2 \pi)$ is the operating frequency.

The apparent permeability can be written as

$\mu=\mu^{\prime}-j \mu^{\prime \prime}$.

So, the real and imaginary parts, deduced from Eq. (9), are expressed as follows:

$$
\begin{aligned}
\mu^{\prime}=1 & +\frac{\omega_{\mathrm{d}}^{2} \chi_{\mathrm{d} 0}\left(\omega_{\mathrm{d}}^{2}-\omega^{2}\right)}{\left(\omega_{\mathrm{d}}^{2}-\omega^{2}\right)^{2}+(\omega \beta)^{2}} \\
& +\frac{\chi_{\mathrm{s} 0} \omega_{\mathrm{s}}^{2}\left(\omega_{\mathrm{s}}^{2}-\omega^{2}+\alpha^{2} \omega^{2}\right)}{\left(\omega_{\mathrm{s}}^{2}-\omega^{2}\left(1+\alpha^{2}\right)\right)^{2}+\left(2 \omega \omega_{\mathrm{s}} \alpha\right)^{2}}, \\
\mu^{\prime \prime}= & \frac{\chi_{\mathrm{d} 0} \omega \beta \omega_{\mathrm{d}}^{2}}{\left(\omega_{\mathrm{d}}^{2}-\omega^{2}\right)^{2}+(\omega \beta)^{2}} \\
& +\frac{\chi_{\mathrm{s} 0} \omega_{\mathrm{s}} \omega \alpha\left(\omega_{\mathrm{s}}^{2}+\omega^{2}\left(1+\alpha^{2}\right)\right)}{\left(\omega_{\mathrm{s}}^{2}-\omega^{2}\left(1+\alpha^{2}\right)\right)^{2}+\left(2 \omega \omega_{\mathrm{s}} \alpha\right)^{2}} .
\end{aligned}
$$


Table 1. Susceptibility dispersion parameters for spin and domain wall resonance of Ferroxcube 3C95 Mn-Zn ferrite.

\begin{tabular}{llllll}
\hline$\chi_{\mathrm{d} 0}$ & $f_{\mathrm{d}}(\mathrm{MHz})$ & $\beta$ & $\chi_{\mathrm{s} 0}$ & $f_{\mathrm{s}}(\mathrm{MHz})$ & $\alpha$ \\
\hline 1400 & $1.4 \times 10^{6}$ & $7.5 \times 10^{6}$ & 900 & $8 \times 10^{6}$ & 5 \\
\hline
\end{tabular}

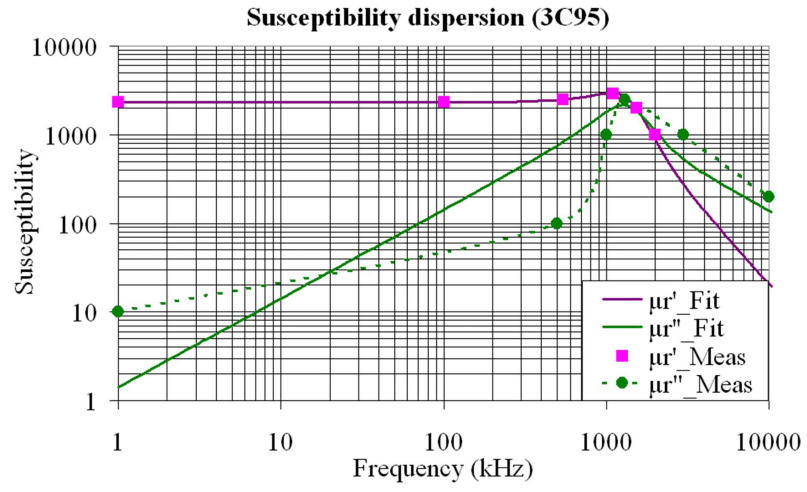

Figure 6. Measured ( $\mu_{\mathrm{r} \_}^{\prime}$ Meas and $\mu_{\mathrm{r} \_}^{\prime \prime}$ Meas) and fitted ( $\mu_{\mathrm{r}-}^{\prime}$ Fit and $\mu_{\mathrm{r}}^{\prime \prime}$ Fit) susceptibility dispersions of 3C95 Mn-Zn ferrite.

The expressions of real and imaginary parts of susceptibilities are quite similar to the one given by Tsutaoka (2003) at a sign near in the numerator of the real component of the susceptibility. We will now consider Mn-Zn ferrite from Ferroxcube of 3C95 type, which appears to be a good candidate for designing an induction sensor thanks to its high relative permeability $\left(\mu_{\mathrm{r}}>2000\right)$, its availability in different shapes and its stability over a wide temperature range (from -100 up to $+200{ }^{\circ} \mathrm{C}$ ). For this material, we have determined on a toroidal core sample the values of the complex susceptibility model parameters $\left(\omega_{\mathrm{d}}, \omega_{\mathrm{s}}, \chi_{\mathrm{d} 0}, \chi_{\mathrm{d} 0}\right.$, and $\left.\omega_{\mathrm{r}}\right)$. These parameters are summarized in Table 1, while the measured and fitted susceptibility dispersions (real and imaginary parts) are plotted in Fig. 6. The obtained values are in the same magnitude range as those reported in Tsutaoka (2003).

\subsection{Complex apparent permeability}

The magnetic gain produced by the ferromagnetic core, known as apparent permeability (Bozorth and Chapin, 1942), allows one to increase the induced voltage. This one results in the combination of the relative permeability of the material $\left(\mu_{\mathrm{r}}\right)$ and its shape, through the demagnetizing coefficient $\left(N_{x, y, z}\right)$ in a given direction $(x, y$ or $z)$. For a long cylinder core of length to diameter ratio $m$, the approximation of the ellipsoid demagnetizing coefficient, given in Osborn (1945), is repeated here:

$N_{z}(m)=\frac{1}{m^{2}}(\ln (2 m)-1)$.

In the current study, a diabolo core shape (shown in Fig. 7) is used, whose apparent permeability ( $\mu_{\text {app }}$ given in Coillot

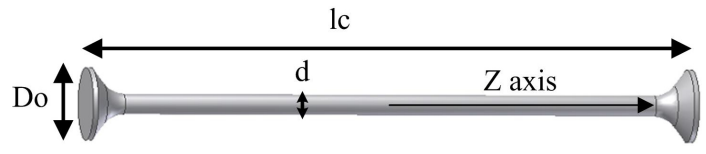

Figure 7. Diabolo core induction sensor.

et al., 2007) is expressed as

$\mu_{\mathrm{app}}=\frac{\mu_{r}}{1+N_{z}(m) \frac{d^{2}}{D_{\mathrm{O}}^{2}}\left(\mu_{r}-1\right)}$,

where $N_{z}\left(m=L_{\mathrm{C}} / D_{\mathrm{O}}\right)$ is the demagnetizing coefficient in the $z$ direction for a cylinder of length $L_{\mathrm{C}}$ and diameter $D_{\mathrm{O}}$.

Assuming that apparent permeability owns real and imaginary parts, it can be written under the following form:

$\mu_{\text {app }}=\mu_{\text {app }}^{\prime}-j \mu_{\text {app }}^{\prime \prime}$.

By substituting, in apparent permeability (Eq. 14), the equation of complex permeability derived for ferrites (Eq. 9), and by identifying it with Eq. (15), we deduce the real and imaginary parts of the apparent complex permeability, respectively Eqs. (16) and (17).

$$
\begin{aligned}
& \mu_{\mathrm{app}}^{\prime}=\frac{\mu^{\prime}\left(1+N_{z}(m) \frac{d^{2}}{D_{\mathrm{O}}^{2}}\left(\mu^{\prime}-1\right)\right)+N_{z}(m) \frac{d^{2}}{D_{\mathrm{O}}^{2}} \mu^{\prime \prime 2}}{\left(1+N_{z}(m) \frac{d^{2}}{D_{\mathrm{O}}^{2}}\left(\mu^{\prime}-1\right)\right)^{2}+\left(N_{z}(m) \frac{d^{2}}{D_{\mathrm{O}}^{2}} \mu^{\prime \prime}\right)^{2}} \\
& \mu_{\mathrm{app}}^{\prime \prime}=\frac{\mu^{\prime \prime}\left(1-N_{z}(m) \frac{d^{2}}{D_{\mathrm{O}}^{2}}\right)}{\left(1+N_{z}(m) \frac{d^{2}}{D_{\mathrm{O}}^{2}}\left(\mu^{\prime}-1\right)\right)^{2}+\left(N_{z}(m) \frac{d^{2}}{D_{\mathrm{O}}^{2}} \mu^{\prime \prime}\right)^{2}}
\end{aligned}
$$

In the case of a ferromagnetic core induction sensor, the inductance equation (Tumanski, 2007) is

$€=\lambda N^{2} \frac{\mu_{0} \mu_{\mathrm{app}} S}{L_{\mathrm{C}}}$,

where $(S)$ is the ferromagnetic core section, $\mu_{0}$ is the vacuum permeability and $\lambda=\left(L_{\mathrm{C}} / L_{\mathrm{W}}\right)^{2 / 5}$ is a correction factor. Thus, the inductance will also have a real part $\left(L^{\prime}\right)$ and an imaginary part $\left(L^{\prime \prime}\right)$ :

$€=L^{\prime}-j L^{\prime \prime}$,

which are written as follows:

$\mathrm{E}^{\prime}=\lambda N^{2} \mu_{0} \frac{\mu_{\text {app }}^{\prime} S}{L_{\mathrm{C}}}$,

$\succeq^{\prime \prime}=\lambda N^{2} \mu_{0} \frac{\left(\mu_{\mathrm{app}}^{\prime \prime}\right) S}{L_{\mathrm{C}}}$. 
Finally, the noise source contribution arising from the ferromagnetic core will look like a Johnson noise whose power spectrum density can be written as

$\operatorname{PSD}_{L}=4 k T \Re(j L \omega)$,

which becomes

$\operatorname{PSD}_{L}=4 k T L^{\prime \prime} \omega$.

In the same way, the mutual inductance will exhibit real and imaginary parts; however, since the mutual inductance is much smaller than the self-inductance, its imaginary part will be neglected and the mutual inductance will be assumed to be a real number.

\section{Modelling and experimental results comparison}

\subsection{The noise equivalent magnetic induction}

The block diagram of Fig. 8 is used to facilitate the computation of the output noise contribution for each noise source. The transmittance of the feedback flux amplifier, given by Eq. (2), is modified to take into account the contribution of the complex inductance:

$T(j \omega)=\frac{V_{\mathrm{OUT}}}{B}=\frac{-j N S G \mu_{\mathrm{app}} \omega}{\left(1-L C \omega^{2}\right)+j \omega\left(\left(R+L^{\prime \prime} \omega\right) C+\frac{G M}{R_{\mathrm{fb}}}\right)}$.

In this block diagram, the noise source coming from the ferromagnetic core is directly added to the thermal noise of the coil resistance. Since this block diagram is dedicated to noise analysis, it is assumed that measured flux $(\varphi)$ is null. For the reasons given in Sect. 3.2, the noise contribution coming from the input resistance of the preamplifier $\left(R_{4}\right)$ is neglected.

The block diagram permits one to determine the transfer function between the output noise contribution (referred to as the $V_{\text {OUT }}$ node) and each of the noise sources. The method is the following: the block diagram is drawn for a given source, while the other noise sources are cancelled thanks to the superposition theorem (for instance, see the block diagram for the feedback resistance noise source shown in Fig. 9).

Then, the closed loop transfer function seen by the $R_{\mathrm{fb}}$ noise is obtained:

$T(j \omega)_{R_{\mathrm{fb}}}=\frac{\frac{j \omega M G}{R_{\mathrm{fb}}}}{1-L^{\prime} C \omega^{2}+j\left(R+L^{\prime \prime} \omega\right) C \omega+\frac{j \omega M G}{R_{\mathrm{fb}}}}$.

Using the general relation between input and output PSD (namely, PSD $_{\mathrm{OUT}}=|T(j \omega)|^{2} \mathrm{PSD}_{\mathrm{IN}}$ ), we deduce the output noise contribution of the feedback resistance:

$\operatorname{PSD}_{R_{\mathrm{fb}}}=4 k T R_{\mathrm{fb}} \frac{\left(\frac{\omega M G}{R_{\mathrm{fb}}}\right)^{2}}{\left(1-L^{\prime} C \omega^{2}\right)^{2}+\left(\left(R+L^{\prime \prime} \omega\right) C \omega+\frac{\omega M G}{R_{\mathrm{fb}}}\right)^{2}}$.

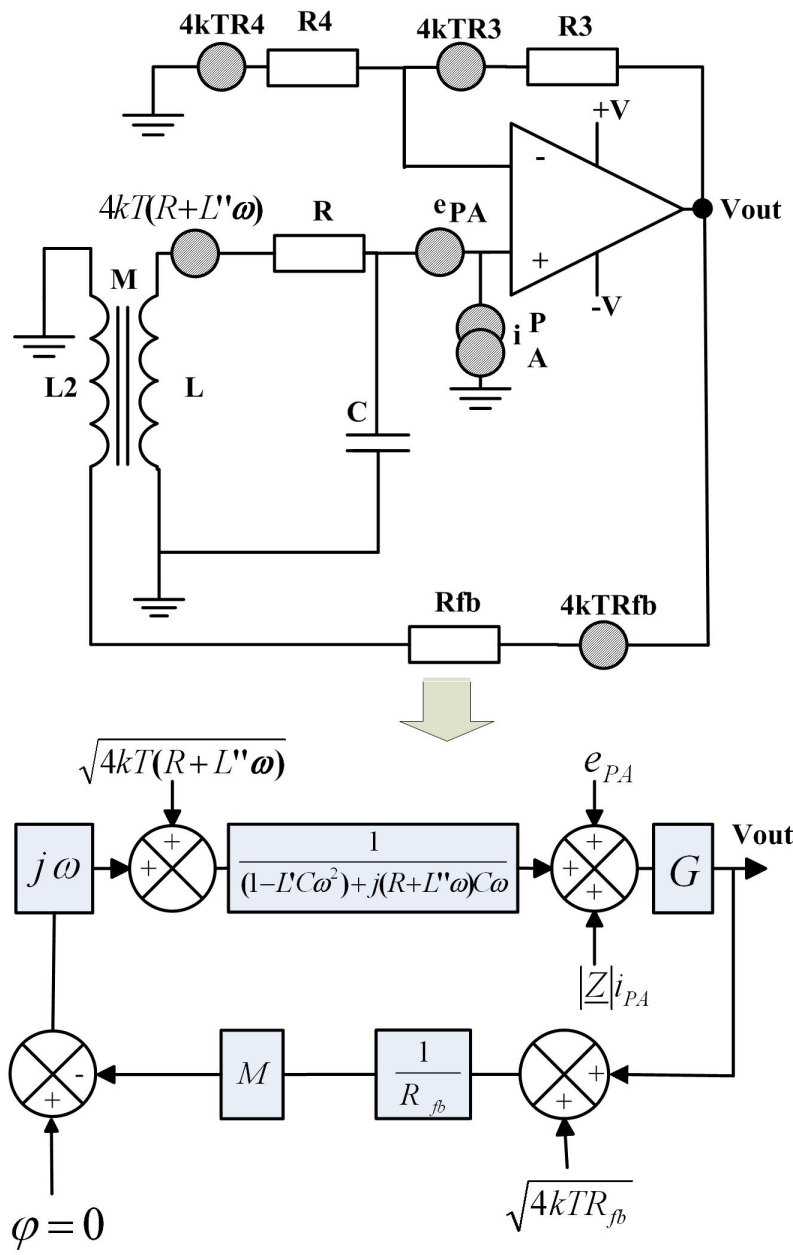

Figure 8. Noise sources in the feedback flux induction configuration and block diagram representation.

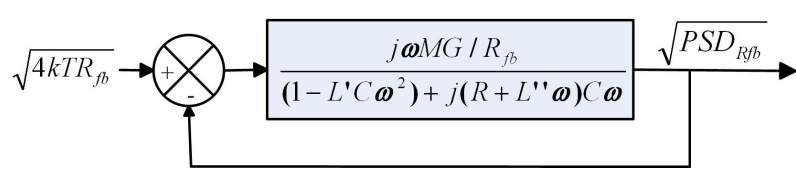

Figure 9. Block diagram representation of the feedback resistance noise source.

This latter expression can be simplified in the frequency range where the feedback flux operates:

$\operatorname{PSD}_{R_{\mathrm{fb}}} \simeq 4 k T R_{\mathrm{fb}}$.

In a similar manner, the noise source contribution from the coil's resistance is derived:

$\operatorname{PSD}_{R}=4 k T \frac{G^{2}\left(R+L^{\prime \prime} \omega\right)}{\left(1-L^{\prime} C \omega^{2}\right)^{2}+\left(\left(R+L^{\prime \prime} \omega\right) C \omega+\frac{G M \omega}{R_{\mathrm{fb}}}\right)^{2}}$. 
The $1 / f$ noise contribution of the preamplifier input voltage noise being neglected, the noise source contribution of the preamplifier input voltage noise is

$$
\operatorname{PSD}_{e_{\mathrm{PA}}}=e_{\mathrm{PA}}^{2} \frac{G^{2}\left(\left(1-L^{\prime} C \omega^{2}\right)^{2}+\left(C \omega\left(R+L^{\prime \prime} \omega\right)\right)^{2}\right)}{\left(1-L^{\prime} C \omega^{2}\right)^{2}+\left(\left(R+L^{\prime \prime} \omega\right) C \omega+\frac{G M \omega}{R_{\mathrm{fb}}}\right)^{2}} .
$$

Similarly, the noise source contribution of the preamplifier input current noise is obtained:

$$
\operatorname{PSD}_{i \mathrm{PA}_{\mathrm{A}}}=\left(|Z| i_{\mathrm{PA}}\right)^{2} \frac{G^{2}\left(\left(1-L^{\prime} C \omega^{2}\right)^{2}+\left(C \omega\left(R+L^{\prime \prime} \omega\right)\right)^{2}\right)}{\left(1-L^{\prime} C \omega^{2}\right)^{2}+\left(\left(R+L^{\prime \prime} \omega\right) C \omega+\frac{G M \omega}{R_{\mathrm{fb}}}\right)^{2}},
$$

where $|Z|$ is the equivalent impedance modulus of the induction sensor seen at the positive input of the amplifier, which is expressed (after some computations) as

$$
|Z|=\sqrt{\frac{\left(\left(R+L^{\prime \prime} \omega+\frac{(M \omega)^{2}}{R_{\mathrm{fb}}}\right)^{2}+\left(L^{\prime} \omega\right)^{2}\right)}{\left(1-L^{\prime} C \omega^{2}\right)^{2}+\left(\left(R+L^{\prime \prime} \omega+\frac{M^{2} \omega^{2}}{R_{\mathrm{fb}}}\right) C \omega+\frac{G M \omega}{R_{\mathrm{fb}}}\right)^{2}}} .
$$

Finally, the total output noise contribution ( $\left.\mathrm{PSD}_{\text {out }}\right)$ is computed by adding the individual power spectral density contribution of each noise source (under the hypothesis of uncorrelated noise):

$$
\mathrm{PSD}_{\text {out }}=\mathrm{PSD}_{Z}+\mathrm{PSD}_{e_{\mathrm{PA}}}+\mathrm{PSD}_{i_{\mathrm{PA}}}+\mathrm{PSD}_{R_{\mathrm{fb}}} .
$$

Finally, the noise equivalent magnetic induction (NEMI), which is the square root of the power spectrum density of the total output noise ( $\mathrm{PSD}_{\mathrm{OUT}}$ ) related to the transfer function modulus of the induction magnetometer $(T(j \omega)$ given by Eq. (24) for feedback flux magnetometer) can be determined.

\subsection{Experimental results and discussion}

A single axis induction magnetometer has been built with an induction sensor using a diabolo core shape made of 3C95 $\mathrm{Mn}-\mathrm{Zn}$ ferrite from Ferroxcube. The sensor has been combined with the MAGIC2 ASIC amplifier. The parameters of the induction sensor design and the preamplifier are summarized in Table 2.

The parameters of the sensor lead to the following value of the electrokinetic modelling: $R=48 \Omega$ (copper wire operating at $300 \mathrm{~K}$ is assumed, i.e. $\left.\rho=1.7 \times 10^{-8} \Omega m\right), L=$ $0.306 \mathrm{H}$ (assuming $\lambda$ equal to 1 ), $M=3 \mathrm{mH}, C=150 \mathrm{pF}$ and $\mu_{\text {app }}=420$. The sensor weight is lower than $30 \mathrm{~g}$, while the ASIC amplifier power consumption supplied with a $12 \mathrm{~V}$ battery is lower than $30 \mathrm{~mW}$. The noise measurement $\left(\mathrm{PSD}_{\text {out }}\right)$ of the induction magnetometer (i.e. sensor connected to its
Table 2. Design parameters.

\begin{tabular}{ll}
\hline Sensor length & $L_{\mathrm{C}}=120 \mathrm{~mm}$ \\
Winding length & $L_{\mathrm{W}}=100 \mathrm{~mm}$ \\
Sensor diameter & $d=4 \mathrm{~mm}$ \\
Diabolo ends diameter & $D_{\mathrm{O}}=14 \mathrm{~mm}$ \\
Turns number & $N=2350$ \\
Feedback coil turns number & $N_{2}=24$ \\
Copper wire diameter & $d_{\mathrm{w}}=0.12 \mathrm{~mm}$ \\
Insulator thickness & $t=25 \mu \mathrm{m}$ \\
Layer number & $n_{1}=4$ \\
Feedback resistance & $R_{\mathrm{fb}}=10 \mathrm{k} \Omega$ \\
Amplifier gain & $G_{\mathrm{dB}}=50.7 \mathrm{~dB}$ \\
Voltage noise & $e_{\mathrm{PA}}=3.3 \mathrm{nV} \sqrt{ }(\mathrm{Hz})^{-1}$ \\
Current noise & $i_{\mathrm{PA}}=20 \mathrm{fA} \sqrt{ }(\mathrm{Hz})^{-1}$ \\
\hline
\end{tabular}

preamplifier) has been performed inside a shielded box consisting of three layers of mu-metal materials and one of conductive material (connected to the preamplifier ground in a way that minimizes the current loop via ground connection. The thickness of each layer is $1 \mathrm{~mm}$ and the inner box side length is $40 \mathrm{~cm}$. Each layer of the shielding box is separated by $1 \mathrm{~cm}$ air gaps (the size of the magnetic shielded box should be much wider than the one of the sensor). The transfer function $(T(j \omega))$ of the induction magnetometer has been measured in gain and phase in a large diameter Helmholtz coil $(1 \mathrm{~m})$ mounted on a wood structure to ensure a homogeneous magnetic field at the scale of the sensor. The accuracy of the facility was verified using a small air-core coil whose theoretical transfer function is fully known. For both measurements (transfer function and noise), an Agilent 35670 spectrum analyser was used. The sensor was equipped with a very thin electrostatic shielding to be insensitive to the electric field component of the electromagnetic waves. The electrostatic shielding was designed to minimize the additional noise from induced current (Ozaki, 2015). The measurements have been done in two configurations: one with the electrostatic shielding and the other one without (in this case, the shielded box plays the role). The simulated NEMI curve, using the modelling of the complex permeability, is compared to the measured one (in the configuration without electrostatic shielding) in Fig. 10.

The result is that the theoretical NEMI (computed for both real and complex permeability) leads to an extremely low minimum NEMI value $\left(<2 \mathrm{fT} \sqrt{ }(\mathrm{Hz})^{-1}\right)$, while practical measurement leads to a higher NEMI value $\left(\sim 4 \mathrm{fT} \sqrt{ }(\mathrm{Hz})^{-1}\right)$ in the same frequency range (namely, 20 and $30 \mathrm{kHz}$ ). The contribution of the complex permeability increases weakly the NEMI (at least in the frequency range between 10 and $100 \mathrm{kHz}$ ). A significant difference between the measured and computed NEMI remains, which suggests that a noise source other than ferromagnetic core contribution dominates and limits the NEMI value in the frequency range where the feedback operates (namely, from 10 to $100 \mathrm{kHz}$ 


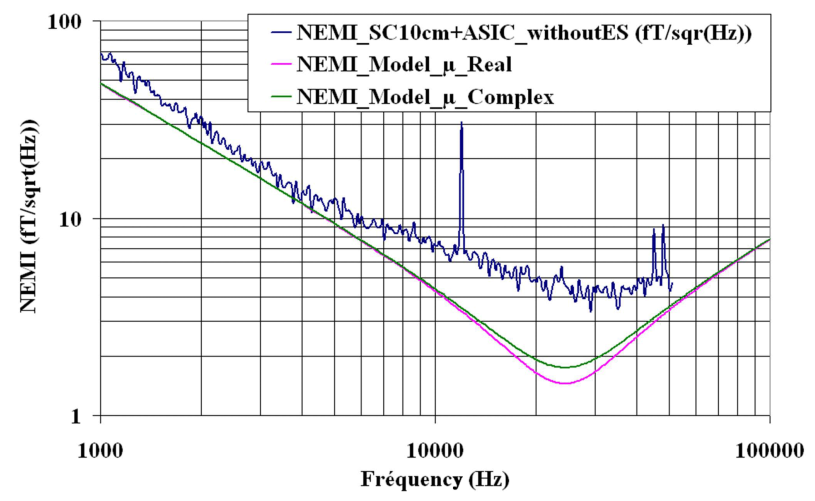

Figure 10. NEMI curves comparison: NEMI with real permeability (pink), NEMI with complex permeability (green) and NEMI measured on a prototype (blue).

in this design). Since the coil was wound directly on the ferromagnetic core, magnetostriction has been suspected of modifying the complex permeability dispersion and thus the higher NEMI measurement. In this aim, a sensor wound on an epoxi tube was realized and a ferrite core with comparable apparent permeability was compared to the sensor reference, but no significant differences have been noticed.

Next, the occurence of an extra noise coming from the coil AC resistance (Butterworth, 1925) is suspected of increasing the Johnson noise contribution coming from the coil resistance (namely, $\mathrm{PSD}_{\mathrm{R}}$ ). The AC resistance increase of the coil comes from the skin effect enhanced by the proximity effect. This effect is taken into account by designers of transformers (Dowell, 1966). In these devices, the AC resistance increase causes extra losses and thus temperature elevation of the transformer. The model proposed by Dowell (1966) is mono-dimensional and assumes a skin depth depending on the distance between wires. However, a lateral skin effect occurring at the end of the winding is also expected (Butterworth, 1925; Belevitch, 1971), making Dowell's model unusable. The contribution of the skin effect enhanced by proximity and the lateral skin effect is also well known to increase strongly the AC resistance and thus to reduce the signal-tonoise ratio of induction sensors for nuclear magnetic resonance (Hoult, 1976). Consequently the skin effect enhanced by the proximity and lateral effect in the case of a multi-layer winding is one of the possible causes which could explain a part of the difference between measurement and modelling.

\section{Conclusions}

While fitting methods usually assume that the extra noise from an induction magnetometer comes from the ferromagnetic core, we have undertaken a modelling attempt of the noise source contribution from a high-permeability $\mathrm{Mn}-\mathrm{Zn}$ ferrite core. The way to take it into account has been achieved by modelling the apparent complex permeability through the susceptibility frequency dispersion of the domain wall motion and magnetization rotation. We have assumed that the machining of the core did not modify the complex permeability. The comparison of the NEMI measurement on a prototype with the NEMI modelling has shown a significant difference around the frequency resonance. Thus, the ferromagnetic core noise seems too weak to explain the difference between the model and the measured NEMI. Thus, the occurrence of an extra noise due to the AC resistance increase is suspected of playing a role. The ferromagnetic core noise contribution (through the apparent complex permeability modelling) should be studied on other ferromagnetic core materials (especially $\mathrm{Ni}-\mathrm{Fe}$ alloy ferromagnetic material). The accurate and rigorous modelling of the NEMI around the resonance frequency remains an issue for fT $\sqrt{\mathrm{Hz}}^{-1}$ induction magnetometer design.

Acknowledgements. The authors would like to thank the reviewers for the time they spent to perform the review and for the stimulating discussions. The authors would also like to thank A. Grosz for the fruitful exchange concerning the delicate NEMI measurements.

Edited by: B. Jakoby

Reviewed by: three anonymous referees

\section{References}

Belevitch, V.: The lateral skin effect in a flat conductor, Philips Tech Rev., 32, 221-231, 1971.

Bin, Y.: An Optimization Method for Induction Magnetometer of $0.1 \mathrm{mHz}$ to $1 \mathrm{kHz}$, IEEE Trans. on Mag., 49, 5294-5300, 2013.

Bozorth, R. M. and Chapin, D.: Demagnetizing factors of rods, J. Appl. Phys., 13, 320-327, 1942.

Butterworth, S.: On the alternating current resistance of solenoidal coils, Proc. R. Soc. Lon. Ser.-A, 107, 693-715, 1925.

Coillot, C., Moutoussamy, J., Leroy, P., Chanteur, G., and Roux, A.: Improvements on the design of search coil magnetometer for space experiments, Sens. Lett., 5, 167-170, 2007.

Coillot, C. and Leroy, P.: Induction Magnetometers: Principle, Modeling and Ways of Improvement, in: Magnetic Sensors - Principles and Applications, edited by: Kuang, K., InTech, ISBN: 978953-51-0232-8, 2012.

Coillot, C., Moutoussamy, J., Boda, M., and Leroy, P.: New ferromagnetic core shapes for induction sensors, J. Sens. Sens. Syst., 3, 1-8, doi:10.5194/jsss-3-1-2014, 2014.

Dosoudil, R., Usakova, M., and Slama, J.: Permeability dispersion in Ni-Zn-Cu ferrite and its composite material, J. Phys., 54, D675-D678, 2004.

Dowell, P. L.: Effects of eddy currents in transformer windings, Proc. the IEEE, 113, 1387-1394, 1966.

Grosz, A., Paperno, E., Amrusi, S., and Liverts, E.: Integration of the electronics and batteries inside the hollow core of a search coil, J. App. Phys., 107, 09E703-1-09E703-3, 2010. 
Hoult, D. I. and Richards, R. E.: The signal-to-noise ratio of the nuclear magnetic resonance experiment, J. Magn. Reson., 24, 71$85,1976$.

Korepanov, V. and Pronenko, V.: Induction Magnetometers: Design Peculiarities, Sens. Transducers J., 120, 92-106, 2010.

Osborn, J. A.: Demagnetizing factors of the general ellipsoids, 67, 351-357, 1945.

Ozaki, M., Yagitani, S., Takahashi, K., and Nagano, I.: DualResonant Search Coil for Natural Electromagnetic Waves in the Near-Earth Environment, IEEE Sens. J., 13, 644-650, 2013.

Ozaki, M., Yagitani, S., Kojima, H., Takahashi, K., and Kitagawa, A.: Current-Sensitive CMOS Preamplifier for Investigating Space Plasma Waves by Magnetic Search Coils, IEEE Sensors Journal, 2, 421-429, 2014.

Ozaki, M., Yagitani, S., Takahashi, K., Imachi, T., Koji, H., and Higashi, R.: Equivalent Circuit Model for the Electric Field Sensitivity of a Magnetic Search Coil of Space Plasma, IEEE Sensors J., 15, 1680-1689, 2015.

Prance, R. J., Clarck, T. D., and Prance, H.: Ultra low noise induction magnetometer for variable temperature operation, Sensor. Actuator., 85, 361-364, 2000.
Rhouni, A., Sou, G., Leroy, P., and Coillot, C.: A Very Low 1/f Noise and Radiation-Hardened CMOS Preamplifier for High Sensitivity Search Coil Magnetometers, IEEE Sens. J., 13, 159$166,2012$.

Ripka, P. (Ed.): Magnetic sensors and magnetometers, Artech House Publ., London, UK, 2000.

Seran, H. C. and Fergeau, P.: An optimized low frequency three axis search coil for space research, Rev. Sci. Instrum., 76, 044502 0044502-9, 2005.

Sobering, T.: Op Amp Noise Analysis, Technnote 5, available at: http://www.k-state.edu/ksuedl/publications/ Technote5-OpampNoiseAnalysis.pdf, 2005.

Tsutaoka, T.: Frequency dispersion of complex permeability in Mn$\mathrm{Zn}$ and $\mathrm{Ni}-\mathrm{Zn}$ spinel ferrites and their composite materials, J. Appl. Phys., 93, 2789-2796, 2003.

Tumanski, S.: Induction coil sensors - A review, Meas Sci. Technol., 18, R31-R46, 2007. 